\title{
Using Boundary Element Method to Calculate the Bending Center of General Section
}

\author{
Xinyan Tang ${ }^{1, a}$ \\ ${ }^{1}$ Engineering College of Nanjing Agricultural University, Nanjing Jiangsu 210031 China
}

\begin{abstract}
Using Siant - Venant bending theory, transverse bending of a general section cylinder, it comes down to solving two boundary integral equations of the same type, the bending function and additional torsion function of the cylinder are obtained. On this basis, using boundary element method to determine the bending center of general section. Finally, to illustrate the application of the method, a numerical example is given.
\end{abstract}

\section{Introduction}

When a solid cylinder with a non-thin wall has a darker missing or a deeper crack, its torsion stiffness will become very small, for example, the circular-section cylinder has a side crack with deep to the center, its torsion stiffness is almost half lost. For this non-thinwalled cylinder with notch or crack, when subjected to horizontal force, apart from the bending deformation of the cylinder, it also causes large additional torsion deformations. To eliminate this additional twist, to improve the bending capacity of the cylinder, methods for processing open thin-walled rods in material mechanics, move transverse force to bend center, that can achieve the goal. In this paper, the theory of Siant Venant[1] is used in the elastic mechanics of the cylinder bending, the problem of transverse bending of a general cylinder, two boundary integral equations of the same type, the bending function and additional torsion function of the cylinder are obtained. Then according to the resulting additional torsion function, the boundary element method can be used to determine the general section of the bending center. To illustrate the application of the method, this paper takes the concave notched rectangular section column as an example, the bending center is calculated by using the boundary element method, variation of torsion stiffness and additional torsion deformation with notch depth. When the concave notch rectangular section is degenerated into a slotted steel type open-walled member, the resulting bending center position is basically consistent with the calculation results of the material mechanics, thus the results were satisfactory.

\section{Bending center of cylinder cross section}

Figure 1 is the cross section of the general cylinder. Inside area is $\Omega, \Gamma$ is boundary, oxy is the centroid spindle for the origin in the cross-sectional center $\bar{c}$.The length of the column is $\mathrm{L}$, the left is properly fixed, right side in the centroid $\bar{c}$ along ox axis act a transverse force $\vec{W}$. Then the cylinder is bent horizontally while, additional torsion of longitudinal axis oz with cross-section around column. In the elastic mechanics, this transverse bending with free torsion is called Saint-Venant bending.

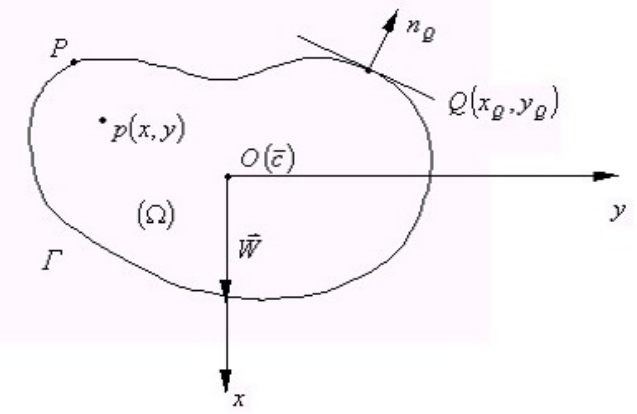

Figure 1. Cross Section of a General Cylinder.

According to the reference[1], this type of bending problem is attributed to finding unknown bending functions $\chi(x, y)$ and unknown additional torsion function $\varphi(x, y)$ defined on a field $\Omega$, they are both planar and harmonic functions. The shear stress on the cross section of the cylinder is determined by the following formula:

$$
\begin{aligned}
& \sigma_{z x}(x, y)=\widetilde{\sigma}_{z x}(x, y)+\bar{\sigma}_{z x}(x, y) \\
& \sigma_{z y}(x, y)=\widetilde{\sigma}_{z y}(x, y)+\bar{\sigma}_{z y}(x, y)
\end{aligned}
$$

\footnotetext{
${ }^{a}$ Corresponding author: tangxiyan@njau.edu.cn
} 
In which, $\tilde{\sigma}_{z x}$ and $\tilde{\sigma}_{z y}$ direct relate to transverse force $\vec{W}$, is called shear stress of bending, they are calculated by the bending function:

$$
\begin{array}{r}
\tilde{\sigma}_{z x}(x, y)=-\mu \beta\left[\frac{\partial \chi}{\partial x}+\frac{v}{2}\left(x^{2}-y^{2}\right)+y^{2}\right] \\
\tilde{\sigma}_{z y}(x, y)=-\mu \beta\left[\frac{\partial \chi}{\partial x}+(2+v) x y\right]
\end{array}
$$

In which, ${ }^{\mu}$ is shear elasticity modulus, $v$ is Poisson's ratio, $\beta=W / E I$ is transverse force coefficient, in which, E is Young's modulus, I is the moment of inertia of the spindle of cross section $\Omega$ around oy:

$$
I=\int_{\Omega} x^{2} \partial \Omega
$$

$\bar{\sigma}_{z x}$ and $\bar{\sigma}_{z y}$ is corresponding to the additional torsion of the cylinder, is called additional twisting shear stress, could be calculated by the function $\varphi(x, y)$ in the following style:

$$
\begin{aligned}
& \bar{\sigma}_{z x}(x, y)=\alpha \mu\left[\frac{\partial \varphi}{\partial x}-y\right] \\
& \bar{\sigma}_{z y}(x, y)=\alpha \mu\left[\frac{\partial \varphi}{\partial y}+x\right]
\end{aligned}
$$

In which, $\alpha$ is additional torsion angle of column unit length, could be called additional twist rate, it is determined by the following formula:

$$
\alpha=\frac{\mu \beta \int_{\Omega}\left[-y \frac{\partial \chi}{\partial x}+x \frac{\partial \chi}{\partial y}-\left(1-\frac{v}{2}\right) y^{3}+\left(2+\frac{v}{2}\right) x^{2} y\right] d \Omega}{D}
$$

In which, $D$ is torsion stiffness of cross section $\Omega$ of the cylinder:

$$
D=\mu \int_{\Omega}\left(x^{2}+y^{2}+x \frac{\partial \varphi}{\partial y}-y \frac{\partial \varphi}{\partial x}\right) d \Omega
$$

According to above shear stress $\left(\bar{\sigma}_{z x}, \bar{\sigma}_{z y}\right)$, can be obtained the additional torque for the section $\Omega$ when the cylinder is laterally bent:

$$
\bar{M}_{K}=\alpha \mu \int_{\Omega}\left(x^{2}+y^{2}+x \frac{\partial \varphi}{\partial y}-y \frac{\partial \varphi}{\partial x}\right) d \Omega
$$

To eliminate additional torsion caused by transverse bending, it is possible to generalize the concept of the bending center of the open thin-walled bar in material mechanics. Move the transverse force $\vec{W}$ in parallel to the ${ }^{o y}$ axis forward one distance $e_{\text {to }}$ the bending center.
Making this force in the section $\Omega$ relative to the centroid $\bar{c}$ produces an internal force torque opposite to the same size of $\bar{M}_{K}$, thereby offsetting the original additional torque, creates a single bend in the cylinder. Therefore, the bending center position $e_{\text {of }}$ the section $\Omega$ is determined by the following formula:

$$
e=\frac{\bar{M}_{K}}{W}
$$

Put (2.10) into the upper type, and note (2.8), so the position of the bend center can be used as a bending function table:

$e=\frac{\int_{\Omega}\left[-y \frac{\partial \chi}{\partial x}+x \frac{\partial \chi}{\partial y}-\left(1-\frac{v}{2}\right) y^{3}+\left(2+\frac{v}{2}\right) x^{2} y\right] d \Omega}{2(1+v) I}$

If you use the plane green formula, the upper table is the integral along the boundary $\Gamma$ of $\Omega$ :

$e=\frac{\left.3 \int_{\Gamma}\left\{x \chi(Q)-\frac{1}{4}\left(1-\frac{v}{2}\right) y^{4}+\left(1+\frac{v}{4}\right) x^{2} y^{2}\right] \cos (n, y)-y \chi(Q) \cos (n, x)\right\} d \Gamma}{2(1+v) \int_{\Gamma} x^{3} \cos (n, x) d \Gamma}$

In addition, torsion stiffness (2.9) can also be represented by boundary integral:

$$
D=\mu \int_{\Gamma}\left\{\left[x \varphi(Q)+\frac{1}{3} y^{3}\right] \cos (n, y)+\left[-y \varphi(Q)+\frac{1}{3} x^{3}\right] \cos (n, x)\right\} d \Gamma
$$

Visible by the above formula, to calculate the bending center position and torsion stiffness of the cylinder cross section $\Omega$ in detail, the boundary values of the bending function and the additional torsion function must be obtained first $\chi(Q)$ and $\varphi(Q)$. This is discussed in the following section.

\section{Boundary integral equation of bending function and additional torsion function}

According to the reference[1], function $\chi(x, y)$ and $\varphi(x, y)$ are all planar harmonic functions on a region $\Omega$, and normal derivative along the outer normal $n_{Q}$ on the boundary $\Gamma$ meet the following boundary conditions, respectively:

$\frac{\partial \chi(x, y)}{\partial n_{Q}}=\frac{\partial \chi(Q)}{\partial n_{Q}}=-\left[\frac{v}{2}\left(x^{2}-y^{2}\right)+y^{2}\right] \cos (n, x)-(2+v) x y \cos (n, y)$ 


$$
\frac{\partial \varphi(x, y)}{\partial n_{Q}}=\frac{\partial \varphi(Q)}{\partial n_{Q}}=y \cos (n, x)-x y \cos (n, y)
$$

In which, $Q=Q(x, y)$ is point on the boundary, $\cos (n, x)$ and $\cos (n, y)$ are direction cosine of outer normal $n_{Q}$ of $\mathrm{Q}$ point.

By the above boundary conditions, could be know, looking for function $\chi(x, y)$ and $\varphi(x, y)$ is two Neumann problems of harmonic function, they can be solved by the boundary integral equation are given directly. In the paper[2], the author has made this introduction, there is no longer a detailed:

$$
\begin{aligned}
& \int_{\Gamma} \chi(Q) \frac{\partial \ln r_{P Q}}{\partial n_{Q}} d \Gamma-\pi \chi(P)=R_{1}(P) \\
& \int_{\Gamma} \varphi(Q) \frac{\partial \ln r_{P Q}}{\partial n_{Q}} d \Gamma-\pi \varphi(P)=R_{2}(P)
\end{aligned}
$$

In which, $r_{P Q}=\sqrt{\left(x_{Q}-x_{P}\right)^{2}+\left(y_{Q}-y_{P}\right)^{2}}$ is the distance between the integration point $Q(x, y)$ on the boundary $\Gamma$ and the reference point $P(x, y)$ on the boundary $\Gamma$, the function to the right of the equation is computed by the known boundary condition (3.1) and (3.2):

$$
\begin{gathered}
R_{1}(P)=\int_{\Gamma} \frac{\partial \chi(Q)}{\partial n_{Q}} \ln r_{P Q} d \Gamma \\
R_{2}(P)=\int_{\Gamma} \frac{\partial \varphi(Q)}{\partial n_{Q}} \ln r_{P Q} d \Gamma
\end{gathered}
$$

Now we only need to solve the above boundary integral equation, the unknown boundary value can be obtained $\chi(Q)$ and $\varphi(Q)$, return them to (2.13) and (2.14), the bending center position $e$ and torsion stiffness $D$ of the cylinder cross section are determined, so the problem was solved. On the solution of boundary integral equation, general use of numerical methods, this paper uses the boundary element method introduced by the author in the paper[3] to solve the numerical problem. On the process of numerical method, it has been described in the paper, not repeat here.

\section{Numerical result}

To illustrate the application of this method, here we use the boundary integral equation given above and the boundary element discrete formula of paper[3]. The bending of a concave notched rectangular column shown in Figure 2 under transverse force $\vec{W}$ is calculated. It's got its torsion stiffness $D$, position of the bending center $e_{\text {and }}$ the additional torsion rate of the cylinder $\alpha$.

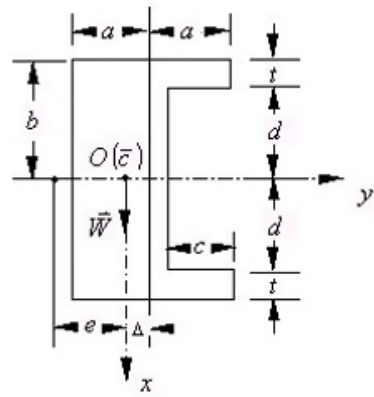

Figure 2. Concave Notched Rectangular Column Section.

In order to compare the results of the open-walled rod with material mechanics, the size ratio of the flange wall thickness $(t)$ to the width $(2 a)$ and height $(2 b)$ of the section in Figure 2 is approximate to the size ratio (9:80:250) of the groove steel $25_{b}$.When numerically calculated, the boundary $\Gamma$ of Figure 2 divided into 130 paragraphs, the calculation is done on the computer. The dimensionless torsion stiffness of the cylinder is $D^{*}=D / D_{0}\left(D_{0}=\mu a^{3} b\right)$, dimensionless bending center distance $e^{*}=e / a$ and dimensionless additional torsion rate is $\alpha / \beta^{*}\left(\beta^{*}=\beta a\right)$, the changes in the dimensionless depth $c^{*}=c / a$ of their concave notch are given by the table 1 below.

In the table $1, \Delta^{*}=\Delta / a, I^{*}=I / I_{0}\left(I_{0}=\frac{4}{3} a b\right)$ are dimensionless centroid position and dimensionless moment of inertia for cylinder cross-section respectively, these parameters are listed to facilitate practical application of the project, the calculation of the centroid

\begin{tabular}{|c|c|c|c|c|c|}
\hline$c^{*}$ & $D^{*}$ & $e^{*}$ & $\alpha / \beta^{*}$ & $\Delta^{*}$ & $I^{*}$ \\
\hline 0. & 4.2615 & 0 . & 0. & 0. & 1. \\
\hline 0.25 & 2.9547 & -0.0307 & -0.3164 & 0.1148 & 0.9001 \\
\hline 0.50 & 1.9214 & -0.0749 & -1.0558 & 0.2266 & 0.8002 \\
\hline 0.75 & 1.1501 & -0.1358 & -2.7995 & 0.3336 & 0.7003 \\
\hline 1.00 & 0.6127 & -0.2212 & -7.3395 & 0.4328 & 0.6004 \\
\hline 1.25 & 0.2750 & -0.3460 & -21.318 & 0.5179 & 0.5005 \\
\hline$\downarrow$ & $\downarrow$ & $\downarrow$ & $\downarrow$ & $\downarrow$ & $\downarrow$ \\
\hline 1.77 & 0.0295 & -0.9623 & -321.59 & 0.5253 & 0.2907 \\
\hline \multicolumn{2}{|c|}{$25_{b}$ groove steel } & \multicolumn{4}{|c|}{$(-1.0568)$} \\
\hline
\end{tabular}
position $\Delta$ is similar to that of the moment of inertia $I$, are all converted from area to boundary $\Gamma$ by Green's formula.

Table 1 
Visible from above table, when the section notch depth c increases, negative movement of bending center to oy axis increased. This indicates that the additional torsion rate $\alpha$ of the cylinder is increased, this is illustrated by the increase in the dimensionless torsion rate $\alpha / \beta^{*}$ in the table. Moreover, when $c^{*}=1.775$, the section of the cylinder in the Figure 2 is degenerated into $25_{b}$ groove steel type open-walled member. At this time, its dimensionless bending center position can also be calculated by the approximate formula of material mechanics. The values are listed in parentheses inside the table, it is basically consistent with the calculation results of this paper, otherwise, when $c=0$, the section of Figure 2 degradation to rectangular section. By this time, the torsion stiffness calculated in this paper is consistent with the theoretical value. It can be seen from this, the method established here is correct, it is suitable for the calculation of the bending center of the general section.

\section{References}

1. Muskhelishvili, N.I., Some Basic Problems of Mathematical Elasticity Mechanics, Science Press, 1958.

2. Xinyan Tang, Torsion Stiffness and Stress Concentration Calculation of Triangular Hole Circular Shaft, Journal of Hohai University, 25 Volumes of Mechanics, 1997.

3. Yiqing Wang, Xinyan Tang, Solving the Torsion Stiffness of Non-Circular Cross-Section Drive Shaft with Boundary Element Method, Journal of Machine Design, 5 Volumes, 1990. 